\title{
DOPED POLY(METHYL METHACRYLATE) PHOTOPOLYMERS FOR HOLOGRAPHIC DATA STORAGE
}

\author{
SHIUAN HUEI LIN*, JUNE-HUA LIN ${ }^{\dagger}$, PO-LIN CHEN ${ }^{\dagger}$, \\ YI -NAN SHIAO $\ddagger$ and KEN Y. HSU ${ }^{\dagger}$ \\ *Department of Electrophysics, National Chiao Tung University, \\ HsinChu, 30050, Taiwan \\ lin@cc.nctu.edu.tw \\ ${ }^{\dagger}$ Institute of Electro-Optical Engineering, \\ National Chiao Tung University, HsinChu, 30050, Taiwan

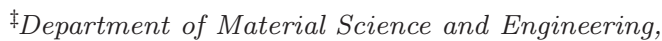 \\ National Chiao Tung University, HsinChu, 30050, Taiwan
}

Received 29 March 2006

\begin{abstract}
In this paper, we report our investigations on thick holographic recording material of the phenanthrenequinone doped poly(methyl methacrylate) (PQ:PMMA) photopolymer. The design strategy and fabrication technique for making thick polymer samples with negligible shrinkage and good optical quality are presented. The physical mechanism for holographic recording in PQ:PMMA material is described, and methods for improving are proposed. Based on these methods, photopolymer samples with different compositions are fabricated and experimentally characterized. The results show that by modifying compositions, the material sensitivity and dynamic range for volume holographic recording have been improved.
\end{abstract}

Keywords: Volume hologram; holographic data storage; holographic material; photopolymer; PMMA polymer.

\section{Introduction}

Holographic data storage has been considered as one of the next generation information storage technologies because of its distinct page oriented data format for parallel data recording/retrieval. ${ }^{1-3}$ It is known that the operation principle of this technology is based on the Bragg condition for thick gratings. In order to achieve high storage density, viz to record multiple holograms in the same spatial location of a storage volume, a recording material of several-millimeter thick and with high photoinduced refractive index change is necessary. Recently, photopolymer materials are of considerable interest for development of write-once material due to their high sensitivity, large modulation in refractive index, and easy fabrication characteristics. ${ }^{4-7}$ The physical mechanism of holographic recording in these materials typically involves photo-induced polymerization of acrylic 
monomers in a polymeric film. A number of photopolymer materials for holographic applications have been developed since the first holographic photopolymer was reported in $1969 .{ }^{8-15}$ However, practical volume holographic materials were not commercially available until recent years when they were announced by companies such as InPhase and Aprilis. The main issues of these kinds of materials are limits in thickness and high shrinkage during holographic exposure. In our laboratory, we have developed a novel technique for fabricating bulk phenanthrenequinone doped poly(methyl methacrylate) (PQ:PMMA) photopolymer of a centimeter thick. ${ }^{16}$ Holographic experiments using these PQ:PMMA samples showed that PMMAbased polymer could be an attractive material due to its good optical quality and negligible photochemical shrinkage. Investigation on the recording mechanism anticipated that by changing the compositions of PMMA-based photopolymers, the performance of holographic recording materials could be further improved. In the following we first review our investigations on PQ:PMMA material. The designing strategy and the fabrication method are described. In Sec. 3, the sensitivity, dynamic range, and reliability for volume holographic recording are defined. These parameters are used to characterize the recording materials. Optical experiments are also presented. Based on these results, we propose some strategies to improve the recording material by changing compositions. Section 4 presents the fabrication and characterization of new PMMA-based photopolymers with different compositions. Experimental results confirm our ideas of improvement. Finally, some conclusions are given in Sec. 5 .

\section{PQ:PMMA Photopolymer Material}

The thick holographic recording material fabricated in our laboratory is PQ:PMMA photopolymer, which consists of the host matrix PMMA doped with PQ molecules. The doped element is photosensitive. Under light illumination, PQ molecules induce the photochemical reaction such that the refractive index of the material changes, and the refractive index pattern follows that of light intensity pattern during holographic recording. This material is suitable for write-once holographic applications. In conventional materials of photopolymerizable PMMA, the fundamental issue is the material shrinkage induced by the photochemical reaction. As a result, the Bragg condition for volume hologram is mis-matched and the recorded information could not be retrieved completely. Our idea for alleviating the shrinkage problem is to make a strong polymer matrix to support the material structure, such that it is not affected by light exposure during holographic recording. Hence, the shrinkage problem can be minimized. On the other hand, in order to record holograms, a small percentage of photosensitive molecules, in our case PQ, are doped and distributed uniformly inside the polymer matrix during material fabrication. These PQ molecules together with a small portion of unreacted monomer molecules are responsible for holographic recording. Thus, the material will be capable of recording phase holograms, whereas the basic photopolymer structure will not be affected by holographic recording. The key to achieving this goal lies in the technique to 
separate the photochemical reaction during holographic recording from the polymerization of the host monomer molecules during material preparation. This can be accomplished if most of the MMA molecules are polymerized to form a host polymer matrix PMMA during material preparation in a way that only a few percentages of unreacted MMA monomer molecules are left for holographic recording.

We have found a two-step thermo-polymerization procedure that can achieve this goal. ${ }^{17}$ In the first step, the thermal initiator, azobisisobutyronitrile (AIBN, $\sim 1 \%$ ) and PQ molecules (up to $0.7 \%$ ) are dissolved in solvent MMA. The solution was stirred in an ultrasonic water bath at $30^{\circ} \mathrm{C}$ for at least $80 \mathrm{hrs}$ until the solution turned homogeneously viscid. In the second step, the solution was poured into a glass container and baked at $45^{\circ} \mathrm{C}$ for $48 \mathrm{hrs}$ until polymerization was complete and the sample became a solid bulk. The actual shape of the sample was determined by the geometry of the glass container. We have found that, careful control of the first step is key to obtaining high quality samples. During this stage, nitrogen gas is released from the thermo-decomposition of AIBN and heat is produced from thermo-polymerization of MMA monomers. In order to produce a bulk sample with no residual air bubbles, the $\mathrm{N}_{2}$ gas molecules and heat should be released completely from the liquid in a slow pace. Thus, this step should be kept at low temperature, in our case room temperature $\left(\sim 25^{\circ} \mathrm{C}\right)$. We have also found that slow stirring is very helpful for releasing $\mathrm{N}_{2}$ gas and heat, thus producing samples with high uniformity.

Our PQ:PMMA samples appear yellow in color. We have measured the optical transmission of different samples in the visible range. The sample possesses strong absorption below the blue wavelength $(<450 \mathrm{~nm})$, and is transparent for red and near infrared wavelengths $(>540 \mathrm{~nm})$. In the holographic experiments we used an argon laser with wavelength $514.5 \mathrm{~nm}$. At this wavelength the absorption coefficient is $2.7 \mathrm{~cm}^{-1}$. We also measured surface flatness of the samples. A disk sample, 5 inches in diameter and $2 \mathrm{~mm}$ thick, was placed into one arm of a Mach-Zehnder interferometer. The interferogram was imaged onto a CCD camera which showed that there was only one fringe across the 5 -inch diameter. This indicates that our technique can produce large samples with high uniformity for holographic data storage application.

\section{Holographic Characteristics of PQ:PMMA Samples}

In general, the desired characteristics for a volume holographic material include high sensitivity for optical exposure, large refractive index change, easy fabrication of large area or volume with high optical quality (or, low scattering noise). From the point of view of holographic data storage, the most important parameters are material sensitivity, dynamic range and reliability.

\subsection{Methodology for characterizing three material parameters}

Optical sensitivity is related to material capability to support data recording speed. High sensitive material enables holographic recording using low power lasers with short exposure time. This is crucial to achieve a compact data storage system with 
high recording speed. There are several ways to define the sensitivity of holographic materials. Basically, they indicate how much exposure energy is required to achieve the requested diffraction efficiency. For holographic data storage, the sensitivity of a material is defined to be equal to the square-root of diffraction efficiency of one hologram divided by the corresponding exposure energy. The square-root value of the diffraction efficiency is named as the grating strength of the hologram.

Dynamic range describes the total change in refractive index of the material that can be produced by optical exposures. In holographic data storage in writeonce material, hundreds or thousands of holograms are multiplexed and recorded at one location of the material until it is photo-chemically exhausted. Each of these holograms shares the refractive index change of this spot. For a specific value of hologram diffraction efficiency, the larger dynamic range of the material means more holograms can be recorded at one spot. The dynamic range of a volume holographic material can be conveniently represented as $M \#=\sum_{i=1}^{N} \sqrt{\eta_{i}}$, where $\eta_{i}$ is diffraction efficiency of each hologram and $N$ is the total number of holograms that can be recorded in the material as it is exhausted. In general, the requested diffraction efficiency $\eta_{i}$ is determined by the sensitivity of the output photodetector array and laser power of the recording beams. A typical value of $\eta_{i}$ is in the order of $10^{-4}$. Thus, the larger dynamic range of the material means the larger the $N$ that can be recorded, resulting in a larger $M \#$ and higher data storage density. The $M$-number is therefore a systemic parameter of the material's dynamic range. ${ }^{18}$

The above two parameters can be experimentally determined by performing multiple plane-wave hologram recording at a single spot in the material. First, a series of holograms were recorded at one location by use of a multiplexing technique until the material was exhausted. Then after each recording, the diffraction efficiency of each hologram was measured, and the summation of the square roots of the diffraction efficiencies formed a running curve of the cumulative grating strength, which is plotted as a function of cumulative exposure energy, viz $C(E)=\sum_{i=1}^{n} \sqrt{\eta_{i}}$, where $E$ is the cumulative exposure energy and $n$ is the total number of holograms that have been recorded by $E$. The curve indicates the dynamics of the build-up process of the multiple hologram recording. When $n$ approaches $N$, the material became exhausted and no more holograms can be recorded, thus $C$ tends to be saturated, and its saturation value is equal to the $M / \#$. On the other hand, according to the definition of material sensitivity, it can be calculated as change of the cumulative grating strength divided by the corresponding exposure energy of that hologram, viz $S=\left.\frac{\Delta C(E)}{\Delta E}\right|_{\text {one hologram }}$, where $\Delta C(E)$ is the grating strength of one hologram with energy $\Delta E$. For a write-once photopolymer in multiple hologram recording, the recording dynamics $C(E)$ will be a nonlinear function of exposure energy $E$. Without loss of generality, we will consider the sensitivity as $S$ calculated for a fresh material, or, when the material is under a few exposures of a multiple storage experiment.

Reliability relates many aspects of volume holographic data storage, such as archival lifetime and temperature stability, etc. For volume holographic storage 


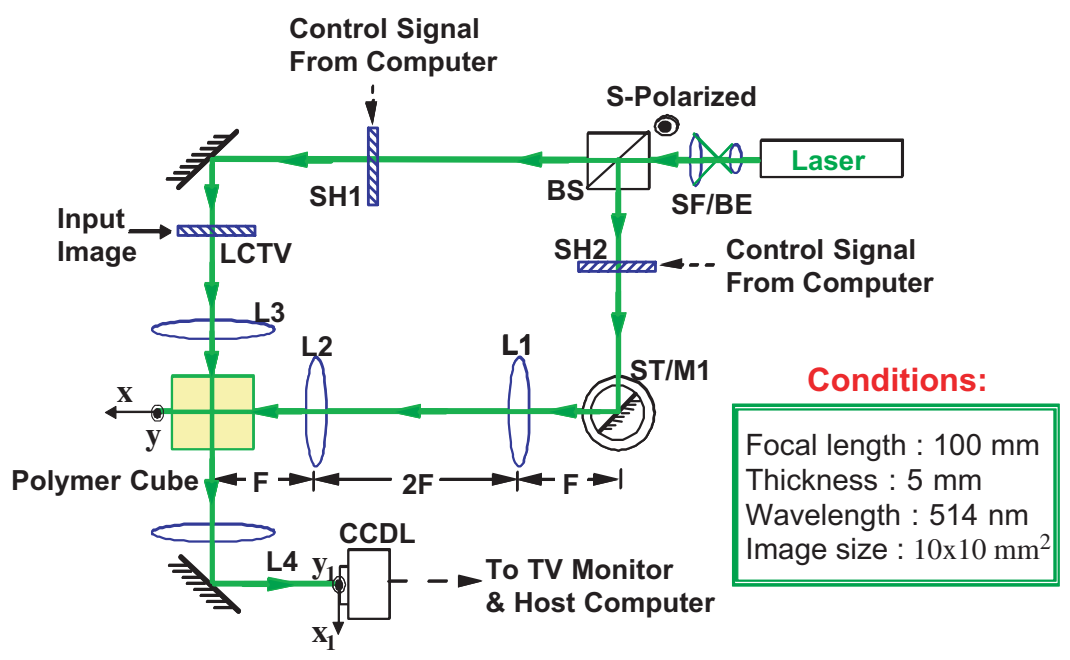

Fig. 1. Schematic diagram of the optical setup to store 250 Fourier holograms using angular multiplexing technique. L1-L4: lens; SH1-SH2: shutter; BS: beam splitter; LCTV: liquid crystal television; SF/BE: spatial filter/beam expander.

using thick materials, good dimensional stability is the most stringent requirement. Photo-chemical reaction induced material shrinkage during holographic recording will result in a mis-match of Bragg condition such that the recorded information cannot be read out completely. ${ }^{19}$ The thicker the material is, the more serious the shrinkage effect. This effect can be evaluated by recording an image into a volume hologram and checking the quality of the retrieved image. Consider a Fourier hologram recording with 90 degrees geometry, where the reference and signal beams are incident into the recording medium at the adjoin sides, as shown in Fig. 1. A photopolymer block with dimensions $5 \times 5 \times 5 \mathrm{~mm}^{3}$ is used as the recording medium. The input image is presented on a LCTV, and the readout image is detected by a CCD camera. The two-dimensional distribution of the reconstructed image can be expressed $\mathrm{as}^{20}$

$$
\begin{aligned}
g\left(x_{1}, y_{1}\right) \propto & f\left(-\frac{1}{\left(1+\alpha_{x}\right)}\left(x_{1}+\frac{\lambda f}{2 \pi} \Delta K_{x}\right),-\frac{1}{\left(1+\alpha_{y}\right)}\left(y_{1}+\frac{\lambda f}{2 \pi} \Delta K_{y}\right)\right) \\
& \times t \operatorname{sinc}\left[\frac { t } { 2 \pi } \left(\Delta K_{z}+\frac{2 \pi}{\lambda f^{2}}\left[x_{1}^{2}+y_{1}^{2}-\left[\frac{\left(1+\alpha_{z}\right)}{\left(1+\alpha_{x}\right)^{2}}\left(x_{1}+\frac{\lambda f}{2 \pi} \Delta K_{x}\right)^{2}\right.\right.\right.\right. \\
& \left.\left.\left.\left.+\frac{\left(1+\alpha_{z}\right)}{\left(1+\alpha_{y}\right)^{2}}\left(y_{1}+\frac{\lambda f}{2 \pi} \Delta K_{y}\right)^{2}\right]+\alpha_{z} f^{2}\right]\right)\right]
\end{aligned}
$$

where $\alpha_{x}, \alpha_{y}$, and $\alpha_{z}$ are material shrinkage coefficients along the $x_{-}, y-$, and $z$-directions, respectively, $\lambda$ is the wavelength, $t$ is the thickness of the medium, $f$ is the focal length of Fourier lenses L3 and L4, and $\Delta K_{x}$ and $\Delta K_{y}$ represent the difference of grating wavevector induced by the shrinkage distortion of hologram. Assume that $t=5 \mathrm{~mm}, f=10 \mathrm{~cm}$, and $\lambda=514.5 \mathrm{~nm}$; then the reconstructed 
Table 1. The reconstructed images under different material shrinkage coefficients (computer simulation). The second row represents the original and reconstructed images with different shrinkage coefficients. The third row represents the envelope profile of the reconstructed image if a plane-wave grating is recorded.

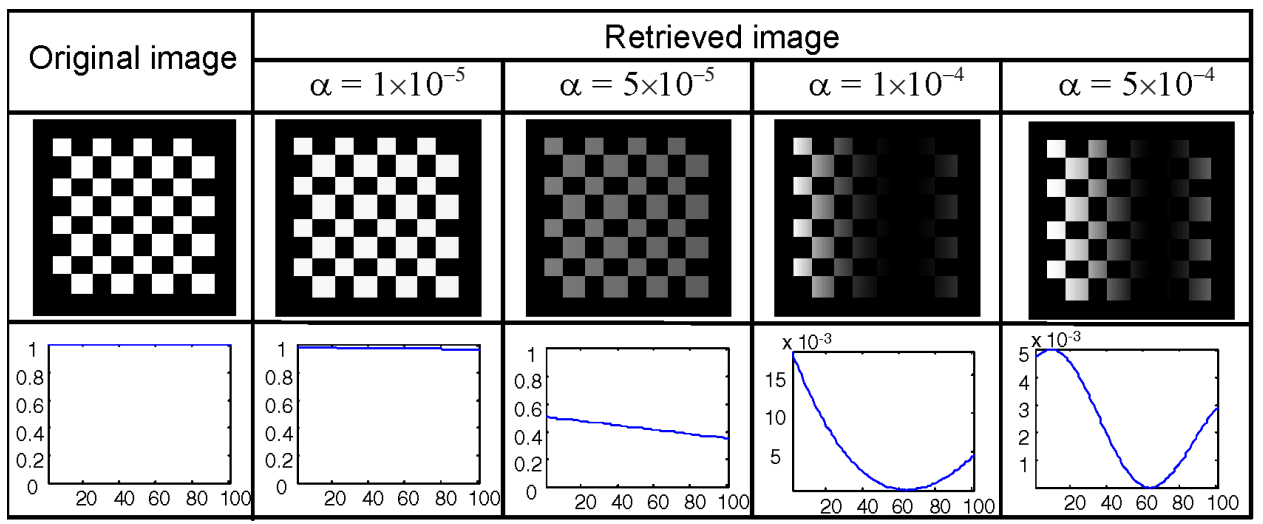

image can be calculated if the shrinkage coefficients are given. Table 1 shows the simulation result with shrinkage coefficient $\alpha=\alpha_{x}=\alpha_{y}=\alpha_{z}$. It can be seen that when the shrinkage coefficient is larger than $5 \times 10^{-5}$, then the retrieved images become seriously distorted. For the 5 -mm thick medium, the shrinkage coefficient should not be larger than $10^{-5}$.

\subsection{Experimental characterization of the material}

In order to measure dynamic range and sensitivity of the material, we have recorded 300 plane-wave holograms on a spot of the material with a peristrophic multiplexing system, each hologram with equal exposure energy $\left(\sim 40 \mathrm{~mJ} / \mathrm{cm}^{2}\right)$. After each recording, the diffraction efficiency of each hologram was measured, and the cumulative grating strength $C(E)$ of the material was plotted as a function of the total exposure energy that has been illuminated on the material during recording. Figure 2 shows the experimental results for photopolymer samples with thickness of $1 \mathrm{~mm}$. If we carry out a curve fitting by the function $C(E)=M \#\left[1-\left(E / E_{\tau}\right)\right]$, then the $M \#$ and the exposure energy constant $E_{\tau}$ of the material are obtained. From these results, sensitivity can be evaluated. As suggested in the previous paragraph, we consider the recording sensitivity of a fresh sample. During this stage, the cumulative exposure energy is small, thus the exponential function $C(E)$ can be linearized and the material sensitivity $S$ can be found to be

$$
\mathrm{S}=\left.\frac{d C(E)}{d E}\right|_{E \rightarrow 0} \approx \frac{M \#}{E_{\tau}} .
$$

From Fig. 2, it is calculated that for the 1-mm thick sample, $M / \#=2.06$ and $E_{\tau}=4.76 \mathrm{~J} / \mathrm{cm}^{2}$. By inserting these values into Eq. (2), the sensitivity of our PQ:PMMA sample is found to be $0.43 \mathrm{~cm}^{2} / J$. The $M \#$ of our PQ:PMMA material 


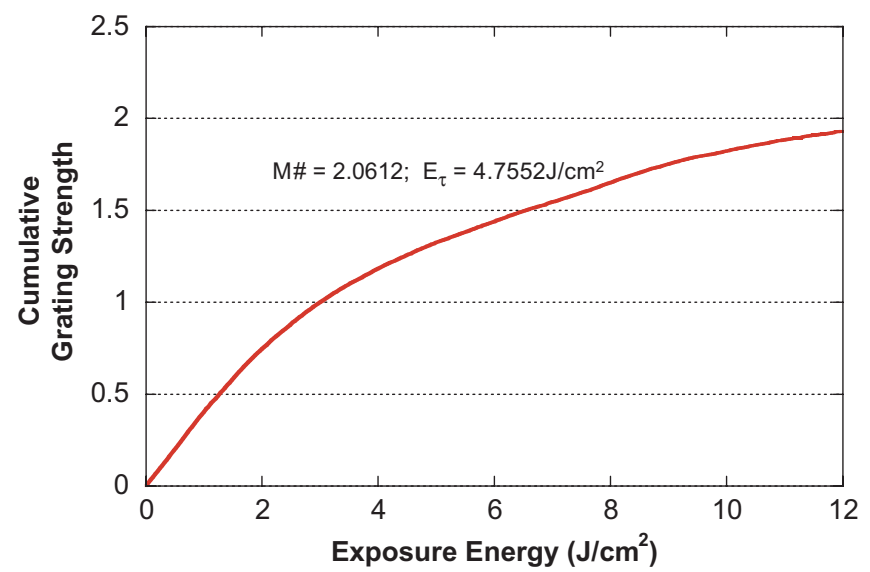

Fig. 2. The running curve of the cumulative grating strength as a function of total exposure energy for a 1-mm thick PQ:PMMA photopolymer sample.

is about comparable with that of $\mathrm{LiNbO}_{3}$ crystals, whereas our material sensitivity is an order of magnitude higher. ${ }^{21}$

For the shrinkage evaluation, we performed a Fourier hologram storage experiment on a $(5 \times 5 \times 5)-\mathrm{mm}^{3}$ photopolymer cube. The schematic diagram of the optical setup is the same as that shown in Fig. 1. The reference and signal beams were incident into the cube at the adjoin sides of the cube. The intensity of each beam was $2 \mathrm{~mW} / \mathrm{cm}^{2}$. An angle-multiplexing scheme was used in the experiment. Two hundred and fifty Fourier holograms of a chessboard pattern, which was shown on a liquid crystal television (LCTV) with resolution of $320 \times 240$ pixels, were recorded on a single location. The original and one of the retrieved images together with the linear scan of the gray level of the corresponding images are shown in Fig. 3. It is found that the reconstructed images have as good fidelity as the original image. Compare the experimental result with that of the analysis presented in the previous paragraph; we can estimate that shrinkage in this material is as low as $10^{-5}$, because the whole image can be reconstructed from such a 5 -mm thick block.

We have investigated possible physical mechanisms for holographic recording in our samples. ${ }^{22}$ Chemical analysis showed that when our photopolymer sample was fabricated, most of MMA monomer was thermally polymerized and only about $10 \%$ of it was left as the residual monomer in bulk samples. At holographic recording, photons excite the o-quinone double bond on the carbonyl functional group of $\mathrm{PQ}$ molecules, and then the $\mathrm{PQ}$ radicals react with the vinyl group on MMA molecules to form one PQ molecule to one MMA molecule attachment. These photocompounds become less conjugated than the original molecular structure such that the refractive index of the sample has been modulated. Consequently, a difference between the refractive index of the polymer matrix in the dark region and that of PQ-MMA compounds in the bright area is created, i.e. a phase grating. In other 

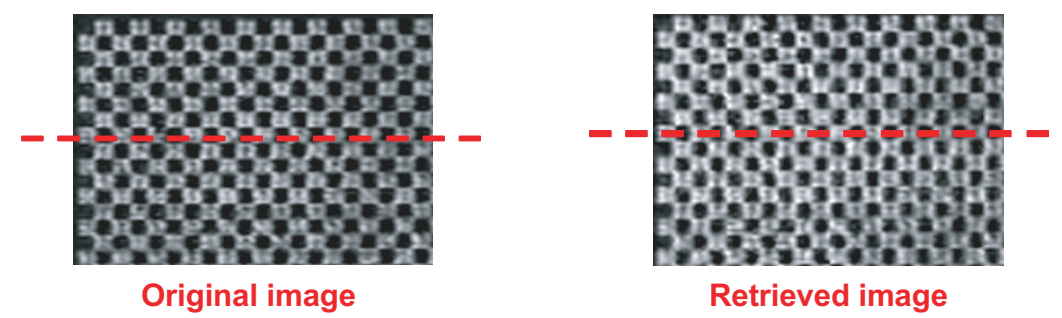

Retrieved image
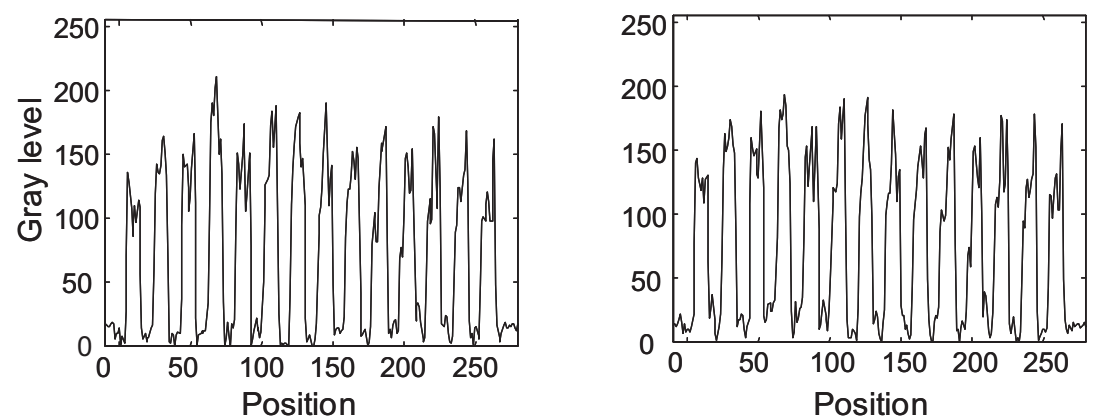

Fig. 3. The original and one of the retrieved images together with the linear scan of the gray level of the corresponding images.

words, hologram recording in our samples is mainly due to refractive index change induced by the structural change of the PQ-MMA attachment. Further, analyses of the molecular weight distribution indicated that the excited PQ molecule also induced a little polymerization of MMA molecules, in which one PQ was linked with four to several units of MMA to form an oligomer. But this effect was relatively small compared to that of PQ-MMA attachment. Such small amounts of the photopolymerization does not affect the backbone of the polymer matrix. Since there is negligible influence on the matrix structure of PMMA chains, thus the volume shrinkage induced by photo-polymerization, which always happens in conventional photopolymer materials, can be minimized.

\section{Improve the Performance of PQ:PMMA Photopolymer}

The above results show that the two-step thermo-polymerization technique is capable of fabricating thick holographic recording material with negligible shrinkage. For the holographic recording, the attachment of the o-quinone double bond on the PQ molecule and the vinyl group on the MMA molecule plays a key role. We can apply this knowledge to further improve material sensitivity and dynamic range of the doped PMMA photopolymer materials. The strategy is to modify the material compositions while maintaining the two-step fabrication technique. Since our PQ:PMMA photopolymer is synthesized from PQ, MMA and AIBN, there are at least three possibilities for improvement. 
The first idea is to play with different photosensitive molecules with o-quinone double bonds. When the side link functional groups of the o-quinone based molecule are modified, the recording sensitivity and dynamic range of the sample may be improved. We have fabricated photopolymer samples with four kinds of doping elements, including the original 9,10-phenanthrenequinone (named as PQ), and 1-isopropyl-7-methyl-9,10-phenanthrenequinone (named as PQ1), 4,5-dinitro9,10-phenanthrenequinone (named as PQ2) and 11,12-dihydrochrysene-11,12-dione (named as PQ3). Table 2 lists their chemical formulas and the corresponding information. Each material was made to $1-\mathrm{mm}$ thick, and doped with the saturated doping concentration of the corresponding photosensitive element, as indicated in Table 2. The holographic recording characteristics of the different samples are measured according to our measuring methodology described in Sec. 3. For PQ and PQ1 doped PMMA samples, three hundred plane-wave holograms, each with equal exposure energy $\left(\sim 40 \mathrm{~mJ} / \mathrm{cm}^{2}\right)$, have been recorded at a single location of the polymer samples. For PQ2 and PQ3 doped PMMA samples, one hundred plane-wave holograms have been recorded, each hologram was recorded with exposure energy of $400 \mathrm{~mJ} / \mathrm{cm}^{2}$ and $800 \mathrm{~mJ} / \mathrm{cm}^{2}$, respectively. The running curves for the cumulative grating strength of different polymer samples are given in Fig. 4. It can be seen that all samples demonstrate the similar recording behavior. By exponentially curve

Table 2. $\quad M \#, \mathrm{E} \tau$ and sensitivity of 1-mm thick PMMA photopolymer samples doped with PQ, PQ1, PQ2 and PQ3, respectively.

Chemical
Formula




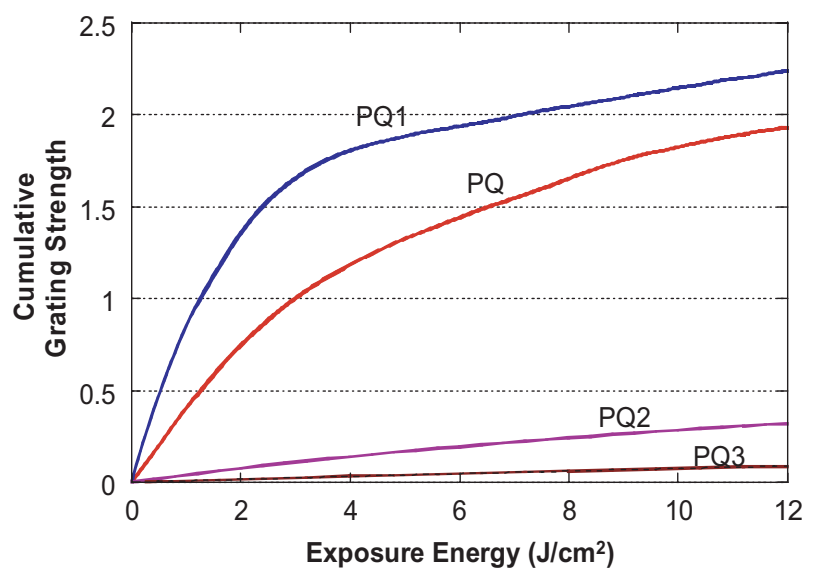

Fig. 4. The running curves of the cumulative grating strength as a function of total exposure energy for 1-mm thick PMMA photopolymer samples doped with PQ, PQ1, PQ2 and PQ3, respectively.

fitting the running curves of Fig. 4, the material $M \#$ and $E_{\tau}$ are estimated and summarized in Table 2. Then, sensitivity of each sample is calculated and listed. It can be seen that the best result is obtained by the PQ1 doped PMMA samples, which has the $M \#$ of $\sim 2.13$ and sensitivity of $1.029 \mathrm{~cm}^{2} / \mathrm{J}$, respectively. The results show that an improvement in both recording sensitivity and dynamic range can be achieved if a suitable dye is chosen.

The second idea is to change the compositions that form the polymer matrix. It can be achieved by increasing the number of vinyl functional groups on the monomer and/or changing the side functional group of the vinyl group on the monomer. The purpose is to enhance the combination ability of monomer molecules with PQ radicals, so as to improve the material dynamic range and sensitivity. Thus, in addition to the original MMA monomer, two kinds of additional monomers, including trimethylolpropane triacrylate (TMPTA) and acrylic acid 2-phenoxyethyl ester (PEA) are added, one at a time. The TMPTA molecule has three vinyl groups, while the PEA molecule has an additional benzene side functional group. Table 3 shows their chemical formulas and the corresponding information. Thus, two types of new photopolymer samples are fabricated. In each type, two monomers are introduced during the fabricating procedure, with a weight-ratio of 2:8 for both TMPTA:MMA and PEA:MMA, respectively. These samples are named as PQ:PTMPTA-co-PMMA and PQ:PPEA-co-PMMA, respectively. The doped concentration of $\mathrm{PQ}$ molecule was fixed at $0.5 \mathrm{wt} \%$ for both samples. For comparison, the PQ:PMMA sample was also made with equal concentration of the PQ molecule. Again, we measured the material $M \#$ and sensitivity for 2-mm thick samples. 175 plane-wave holograms, each with the same exposure energy $\left(\sim 0.6 \mathrm{~J} / \mathrm{cm}^{2}\right)$, were recorded at a single location of the sample. The running curves of the cumulative 
Table 3. $M \#, \quad E_{\tau}$ and sensitivity of 2-mm thick PQ/PTMPTA-co-PMMA and PQ/PPEA-co-PMMA photopolymer samples.

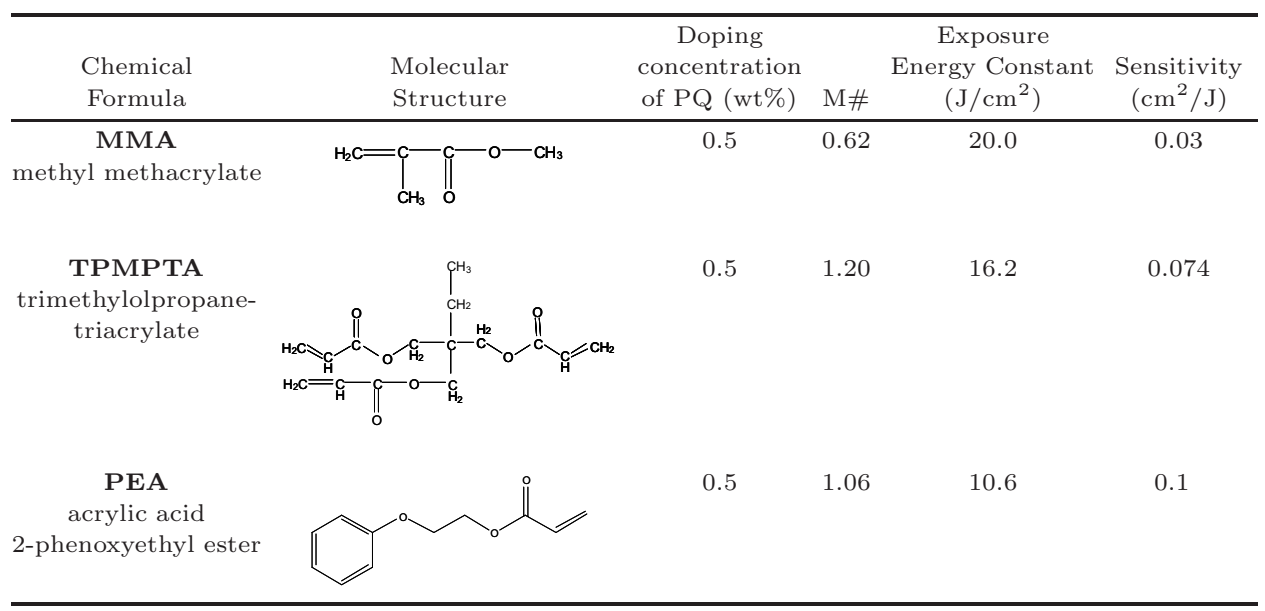

grating strength for different samples are shown in Fig. 5. Curve fittings are performed and the $M \#, E_{\tau}$, and $S$ are calculated and listed in Table 3 . It is seen in the table that the $M / \#$ of PQ:PPEA-co-PMMA is 1.06 , which is 1.5 times larger than that of PQ:PMMA (M\# 0.62). On the other hand, the dynamic range of PQ:PTMPTA-co-PMMA has been improved by about 2 times to become 1.20. Furthermore, the exposure energy constant, $E_{\tau}$ of PQ/PTMPTA-co-PMMA and PQ/PPEA-co-PMMA are $16.21 \mathrm{~J} / \mathrm{cm}^{2}$ and $10.60 \mathrm{~J} / \mathrm{cm}^{2}$, respectively. Both are slightly lower than that of PQ/PMMA $\left(E_{\tau} \sim 20 \mathrm{~J} / \mathrm{cm}^{2}\right)$. Using these parameters, sensitivity can be calculated, as shown in last column in Table 3. It can be seen

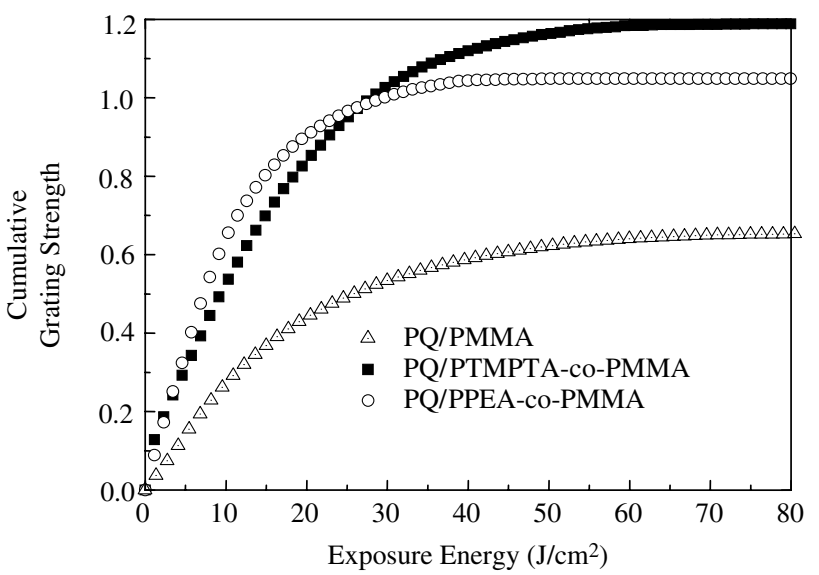

Fig. 5. The running curves of the cumulative grating strength as a function of total exposure energy for 2-mm thick PQ/PTMPTA-co-PMMA and PQ/PPEA-co-PMMA photopolymer samples. 
that $S$ has been improved by about 2 and 3 times, respectively. The results confirm our idea that by adding other components to form a copolymer structure, the holographic recording characteristics of PQ:PMMA photopolymer can be improved.

The third alternative for improving PQ:PMMA material is to add another doping element, so as to provide a catalyst to speed up the photoreaction between the PQ and MMA molecules. Zinc methacrylate (ZnMA) has been chosen in our investigation. It was added up to $0.35 \mathrm{wt} \%$ into PQ:PMMA photopolymer to make ZnMA and PQ co-doped PMMA samples with thickness of $2 \mathrm{~mm}$, which is named as ZnMA/PQ:PMMA. In order to measure the material $M \#$ and sensitivity, 175 plane-wave holograms were recorded at one location of the material, each with equal exposure energy $\left(\sim 0.31 \mathrm{~J} / \mathrm{cm}^{2}\right)$. Figure 6 shows the running curves for photopolymer samples with different doping concentrations of ZnMA molecules. From this figure, the $M \#, E_{\tau}$ and $S$ for the different samples are estimated and summarized in Table 4. It shows that when the doping concentration of ZnMA molecule is increased from 0 to $0.35 \mathrm{wt} \%$, the $M / \#$ is increased by about 3 times, from 2.83 to 8.81, and the exposure energy constant is changed slightly. As a result, the sensitivity is improved by 2.5 times $\left(1.23 \mathrm{~cm}^{2} / \mathrm{J}\right.$ for $\left.0.35 \mathrm{wt} \% \mathrm{ZnMA}\right)$, compared

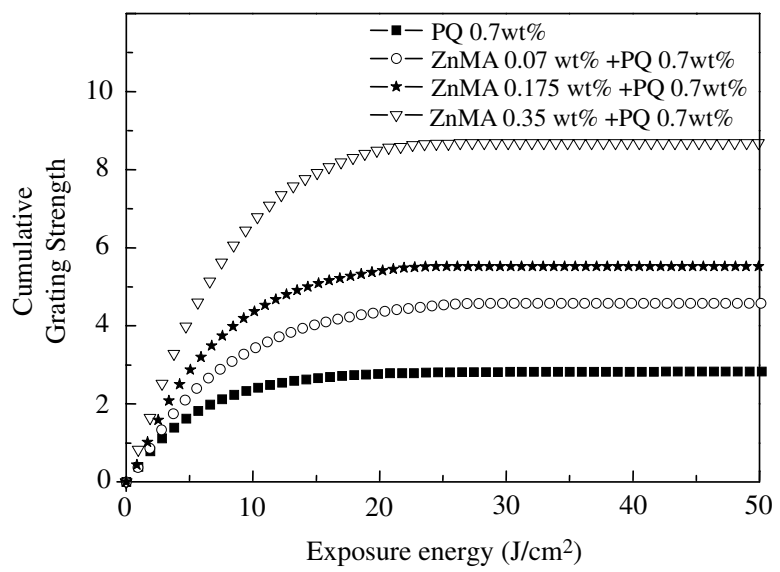

Fig. 6. The running curves of the cumulative grating strength as a function of total exposure energy for PQ/ZnMA:PMMA photopolymer samples with different concentrations of ZnMA molecule. Notations on the right corner indicate the doping concentrations of ZnMA and PQ molecules, respectively.

Table 4. $M \#, E_{\tau}$, and sensitivity of $2-\mathrm{mm}$ thick PQ/ZnMA: PMMA photopolymer samples with different concentrations of ZnMA molecule.

\begin{tabular}{lcccc}
\hline Conc. ZnMA (wt\%) & 0 & 0.07 & 0.175 & 0.35 \\
\hline $\mathrm{M \#}$ & 2.83 & 4.63 & 5.59 & 8.81 \\
$\mathrm{E}_{\tau}\left(\mathrm{J} / \mathrm{cm}^{2}\right)$ & 5.53 & 7.49 & 6.75 & 7.15 \\
Sensitivity $\left(\mathrm{cm}^{2} / \mathrm{J}\right)$ & 0.51 & 0.62 & 0.83 & 1.23 \\
\hline
\end{tabular}


with that of the PQ singly-doped PMMA sample $\left(0.51 \mathrm{~cm}^{2} / \mathrm{J}\right)$. These experimental results demonstrate that by adding the catalyst type molecules, such as ZnMA, into PQ:PMMA photopolymer, it is possible to improve both the $M \#$ and sensitivity of the material for volume holographic recording.

\section{Conclusions}

We have presented our investigations on the holographic recordings in doped poly(methyl methacrylate) photopolymers. The design strategy and the fabrication technique for making thick polymer samples with negligible shrinkage and good optical quality are described. The physical mechanism for holographic recording in this material is presented. In terms of material dynamic range and sensitivity, a methodology for characterizing the material for holographic data storage has been proposed. For the PQ:PMMA samples, the shrinkage coefficient of the samples is estimated to be smaller than $10^{-5}$, the $M / \#$ of a 1 -mm thick sample is measured to be 2.06 , and the sensitivity is calculated to be $0.43 \mathrm{~cm}^{2} / \mathrm{J}$. By modifying the components in materials, we then presented three possibilities to improve the material. The first method is to fabricate a photopolymer doped with different photosensitive dye molecules. The best result is achieved with a PQ1:PMMA sample, of which the $M \#$ of the 1-mm thick sample is slightly improved, and the sensitivity is about 2.4 times higher than that of PQ:PMMA sample. The second method is to fabricate a photopolymer sample with PTPMPTA-co-PMMA or PPEA-co-PMMA copolymers. The $M \#$ have been improved by $1.5 \sim 2$ times, and the sensitivity of these materials has been improved by $2 \sim 3$ times. The third idea is to add catalyst molecules, such as ZnMA, into the composition to form a co-doped photopolymer so that the photo-chemical reaction can be speeded up. The material $M \#$ of the ZnMA/PQ:PMMA sample was measured to have improved by 3 times and the material sensitivity by 2.5 times. The above results have confirmed our idea of changing material composition for enhancing the holographic recording characteristics. However, they are by no means the optimal conditions yet. We anticipate that by suitable selection of photosensitizers, co-doped monomer molecules and catalyst molecules, the material dynamic range and sensitivity can be further improved.

\section{Acknowledgment}

The authors are grateful for helpful discussion about material preparation from Prof. Wha Tzong Whang. We gratefully acknowledge the financial support from the National Science Council, Taiwan under contracts 89-E-FA06-1-4 and NSC922112-M-009-010.

\section{References}

1. L. K. Anderson, Bell Laboratories Record 45 (1968) 319-326.

2. J. Heanue, M. Bashaw and L. Hesselink, Science 265 (1994) 749-752. 
3. D. Psaltis and F. H. Mok, Sci. Am. 273(5) (1995) 70-76.

-4. A. Pu and D. Psaltis, Appl. Opt. 35 (1996) 2389-2398.

5. V. L. Colvin, R. G. Larson, A. Haris and M. L. Shilling, J. Appl. Phys. 81 (1997) 5914-5923.

6. H. Ono, T. Tamoto, A. Emoto and N. Kawatsuki, Jap. J. Appl. Phys. 44 (2005) $1783-1788$.

7. U. V. Mahilny, D. N. Marmysh, A. I. Stankevich, A. L. Tolstik, V. Matusevich and R. Kowarschik, Appl. Phys. B 82(2) (2006) 299-302.

8. D. H. Close, A. D. Jacobson, J. D. Margerum, R. G. Brault and F. J. McClung, Appl. Phys. Lett. 14 (1969) 159.

9. R. A. Lessard and G. Manivannan (eds.), Selected Papers on Photopolymers, MS 114 (SPIE Opt. Emg. Press, Bellingham, Washington, 1995)

10. D. J. Lougnot, Crit. Rev. Opt. Sci. Technol. CR63 (1996) 190-213.

11. S. H. Stevenson, Proc. SPIE 3011 (11997) 231-241.

12. P. Chebena and M. L. Calvo, Appl. Phys. Lett. 78(11) (2001) 1490-1492.

13. D. A. Waldman, H. Y. S. Li and M. G. Horner, J. Imag. Sci. Tech. 41(5) (1997) 497-514.

14. L. Dhar, A. Hale, H. E. Katz, M. L. Schilling, M. G. Schnoes and F. C. Schilling, Opt. Lett. 24(7) (1999) 487-489.

15. H. J. Coufal, D. Psaltis and G. T. Sincerbox, Holographic Data Storage, Part II (Springer, New York, 2000), pp. 172-208.

16. S. H. Lin, K. Y. Hsu, W. Z. Chen and W. T. Whang, Opt. Lett. 25(7) (2000) 451-453.

17. K. Y. Hsu, S. H. Lin, Y. N. Hsiao and W. T. Whang, Opt. Eng. 42(5) (2003) 1390-1396.

18. F. Mok, G. Burr, and D. Psaltis, Opt. Lett. 21(12) (1996) 896-898.

19. M. L. Hsieh and K. Y. Hsu, Opt. Eng. 40(10) (2001) 2125-2133.

20. K. Y. Hsu and S. H. Lin, Proc. SPIE 5206 (2003) 142-148.

21. H. Günther, R. Macfarlane, Y. Furukawa, K. Kitamura and R. Neurgaonkar, Appl. Opt. 37 (1998) 7611-7623.

22. Y.-N. Hsiao, W.-T. Whang and S. H. Lin, Opt. Eng. 43(9) (2004) 1993-2002. 


\section{This article has been cited by:}

1. Chengmingyue Li, Liangcai Cao, Qingsheng He, Guofan Jin. 2014. Holographic kinetics for mixed volume gratings in gold nanoparticles doped photopolymer. Optics Express 22:5, 5017. [CrossRef]

2. Shiuan Huei Lin, Sheng-Lung Cho, June-Hua Lin, Ken Y. Hsu, Sien Chi. 2014. Influence of fabrication conditions on characteristics of phenanthrenequinone-doped poly(methyl methacrylate) photopolymer for holographic memory. Optics Communications . [CrossRef]

3. A. V. Veniaminov, U. V. Mahilny. 2013. Holographic polymer materials with diffusion development: Principles, arrangement, investigation, and applications. Optics and Spectroscopy 115:6, 906-930. [CrossRef]

4. Mei-Li Hsieh, Wei-Cheng Chen, Hong-Yu Chen, Shawn-Yu Lin. 2013. Optimization of light diffraction efficiency and its enhancement from a doped-PMMA volume holographic material. Optics Communications 308, 121-124. [CrossRef]

5. Shiuan Huei Lin, Po-Lin Chen, Chun-I Chuang, Yu-Faye Chao, Ken Y. Hsu. 2011. Volume polarization holographic recording in thick phenanthrenequinone-doped poly(methyl methacrylate) photopolymer. Optics Letters 36:16, 3039. [CrossRef]

6. Chun-I. Chuang, Yi-Nan Hsiao, Shiuan-Huei Lin, Yu-Faye Chao. 2010. Real-time measurement of photo-induced effects in 9,10-phenanthrenequinone-doped poly(methyl methacrylate) photopolymer by phase-modulated ellipsometry. Optics Communications 283:17, 3279-3283. [CrossRef]

7. Jian Wang, Xiudong Sun, Suhua Luo, Yongyuan Jiang. 2010. Study on the mechanism of dark enhancement in phenanthrenequinone-doped poly(methyl methacrylate) photopolymer for holographic recording. Optics Communications 283:9, 1707-1710. [CrossRef]

8. Jian Wang, Xiudong Sun, Suhua Luo, Yongyuan Jiang. 2009. The shift of Bragg angular selectivity curve in darkness in glass-like photopolymer for holographic recording. Optical Materials 32:1, 261-265. [CrossRef]

9. Jyh-Herng Chen, Chin-Tien Yang, Chao-Hui Huang, Ming-Fang Hsu, Tzuan-Ren Jeng. 2009. Study of Optical Properties of Glass-Like Polymer Material for Blue Laser Holographic Optic Data Storage Recording. IEEE Transactions on Magnetics 45:5, 2256-2259. [CrossRef]

10. Jyh-Herng Chen, Chin-Tien Yang, Chong-Chieh Cheng, Ming-Fang Hsu, Tzuan-Ren Jeng. 2009. Effect of Photosensitizers on the Shrinkage Characteristics of Blue Laser Sensitive Holographic Epoxy Materials. IEEE Transactions on Magnetics 45:5, 2252-2255. [CrossRef]

11. S H Lin, Y-N Hsiao, K Y Hsu. 2009. Preparation and characterization of Irgacure 784 doped photopolymers for holographic data storage at $532 \mathrm{~nm}$. Journal of Optics A: Pure and Applied Optics 11:2, 024012. [CrossRef]

12. Shiuan Huei Lin, Po-Lin Chen, June-Hwa Lin. 2009. Phenanthrenequinone-doped copolymers for holographic data storage. Optical Engineering 48:3, 035802. [CrossRef]

13. Balázs Gombkötő, Zsolt Nagy, Pál Koppa, Emőke Lőrincz. 2008. Modeling high density microholographic data storage: Using linear, quadratic, thresholding and hard clipping material characteristics. Optics Communications 281:17, 4261-4267. [CrossRef]

14. S. -H. Lin, Y. -N. Hsiao, K. Y. Hsu, W. -T. Whang. 2008. Eosin Y spirit soluble/Triethanolamine doped poly (methyl methacrylate-co-hydroxyethyl methacrylate) photopolymer for holographic data storage. Optical Memory and Neural Networks 17:2, 119-125. [CrossRef] 
15. Shiuan-Huei Lin, Po-Lin Chen, Yi-Nan Hsiao, Wha-Tzong Whang. 2008. Fabrication and characterization of poly(methyl methacrylate) photopolymer doped with 9,10phenanthrenequinone (PQ) based derivatives for volume holographic data storage. Optics Communications 281:4, 559-566. [CrossRef] 